\title{
Molecular characterization of a long range haplotype affecting protein yield and mastitis susceptibility in Norwegian Red cattle
}

Marte Sodeland ${ }^{1 *}$, Harald Grove ${ }^{1}$, Matthew Kent ${ }^{1}$, Simon Taylor ${ }^{1}$, Morten Svendsen $^{2}$, Ben J Hayes ${ }^{1,3}$ and Sigbjørn Lien ${ }^{1}$

\begin{abstract}
Background: Previous fine mapping studies in Norwegian Red cattle (NRC) in the region 86-90.4 Mb on Bos taurus chromosome 6 (BTA6) has revealed a quantitative trait locus (QTL) for protein yield (PY) around $88 \mathrm{Mb}$ and a QTL for clinical mastitis (CM) around $90 \mathrm{Mb}$. The close proximity of these QTLs may partly explain the unfavorable genetic correlation between these two traits in NRC. A long range haplotype covering this region was introduced into the NRC population through the importation of a Holstein-Friesian bull (1606 Frasse) from Sweden in the 1970s. It has been suggested that this haplotype has a favorable effect on milk protein content but an unfavorable effect on mastitis susceptibility. Selective breeding for milk production traits is likely to have increased the frequency of this haplotype in the NRC population.

Results: Association mapping for PY and CM in NRC was performed using genotypes from 556 SNPs throughout the region 86-97 Mb on BTA6 and daughter-yield-deviations (DYDs) from 2601 bulls made available from the Norwegian dairy herd recording system. Highest test scores for PY were found for single-nucleotide polymorphisms (SNPS) within and surrounding the genes CSN2 and CSN1S2, coding for the $\beta$-casein and $\alpha_{S_{2} \text {-casein }}$ proteins. High coverage re-sequencing by high throughput sequencing technology enabled molecular characterization of a long range haplotype from 1606 Frasse encompassing these two genes. Haplotype analysis of a large number of descendants from this bull indicated that the haplotype was not markedly disrupted by recombination in this region. The haplotype was associated with both increased milk protein content and increased susceptibility to mastitis, which might explain parts of the observed genetic correlation between PY and CM in NRC. Plausible causal polymorphisms affecting PY were detected in the promoter region and in the 5'-

flanking UTR of CSN1S2. These polymorphisms could affect transcription or translation of CSN1S2 and thereby affect the amount of $\alpha_{S_{2}}$-casein in milk.

Highest test scores for CM were found in the region 89-91 Mb on BTA6, very close to a cluster of genes coding for CXC chemokines. Expression levels of some of these CXC chemokines have previously been shown to increase in bovine mammary gland cell lines after exposure to bacterial cell wall components.

Conclusion: Molecular characterization of the long range haplotype from the Holstein-Friesian bull 1606 Frasse, imported into NRC in the 1970s, revealed polymorphisms that could affect transcription or translation of the casein gene CSN1S2. Sires with this haplotype had daughters with significantly elevated milk protein content and selection for milk production traits is likely to have increased the frequency of this haplotype in the NRC population. The haplotype was also associated with increased mastitis susceptibility, which might explain parts of the genetic correlation between PY and CM in NRC.
\end{abstract}

\footnotetext{
* Correspondence: marte.sodeland@umb.no

${ }^{1}$ Centre for Integrative Genetics, Department of Animal and Aquacultural

Sciences, Norwegian University of Life Sciences, N-1432 Aas, Norway

Full list of author information is available at the end of the article
} 


\section{Background}

An unfavourable genetic correlation between PY and clinical mastitis $(\mathrm{CM})$ has been reported in Norwegian Red cattle (NRC), with estimates ranging from 0.21 to 0.55 [1]. This genetic correlation could be both due to pleiotropic effects and to QTLs affecting the two traits being closely positioned on bovine chromosomes. The heritability for PY is estimated to be 0.19 in NRC [1], and is higher than the heritability for $\mathrm{CM}$ for which estimates range between 0.02 and 0.12 in Nordic cattle populations [2-4]. CM is inflammation of the mammary gland and the most costly disease affecting dairy cattle world-wide. In addition to economical considerations the disease also affects animal welfare. Milk production traits were included in the breeding goal for NRC earlier than health traits were, and selective breeding for PY could have increased the frequency of variants with undesirable effects on CM.

It has been suggested by Lien et al. [5] that a haplotype encompassing the casein gene cluster around 88 $\mathrm{Mb}$ on Bos taurus chromosome 6 (BTA6), which confers a favorable effect on milk production traits, was introduced into the NRC population through the importation of a Swedish Holstein-Friesian bull (1606 Frasse) in the 1970s. This bull has had a major influence on NRC, with an estimated contribution to this population above $8 \%$ (Svendsen, personal communication). Frasse 1606 and his sons were favored due to high breeding values for milk production traits before animal health traits were included in NRC breeding schemes, and might have contributed to generating a positive correlation between PY and CM in this population.

Association mapping has revealed that a quantitative trait locus (QTL) for protein yield (PY) coincides with the casein gene cluster [6-10]. Casein proteins constitute approximately $80 \%$ of dairy cattle milk protein and polymorphisms in these genes have been shown to contain variation associated with milk protein composition and protein content in other populations $[5,7,11-16]$. It has further been reported that the haplotype from 1606 Frasse encompassing the casein gene cluster is associated with increased mastitis susceptibility, and a QTL for $\mathrm{CM}$ in the periparturient period has been found around $90 \mathrm{Mb}$, close to the QTL affecting PY around 88 $\mathrm{Mb}$ [6]. Taken together these results suggest that causal polymorphism(s) residing within this genomic region may be influencing these two important traits.

Opportunities for fine mapping and molecular characterization of QTL regions have been improved by recent developments in high throughput sequencing and genotyping technologies [17-21], and it is well established that genotyping of related animals with well documented pedigree increases the accuracy of haplotyping and imputation methods $[22,23]$. Accuracy of imputation and power of association mapping using imputed genotypes is increased in populations with extensive linkage disequilibrium (LD) [24-27], meaning that a combination of these approaches is a feasible strategy in cattle populations [28-30].

Aims of this study were to refine the location of causative polymorphisms on BTA6 affecting PY and CM, and perform molecular characterization of the 1606 Frasse haplotype by re-sequencing in order to identify the most plausible causative polymorphism(s) underlying the two QTLs.

\section{Results and discussion}

Re-sequencing in the genomic region between 86 and $97 \mathrm{Mb}$ on BTA6 was done by first capturing sequence from seven genomic DNA samples using a Nimblegen sequence capture array, and then sequencing the product on a Roche 454 GS-FLX sequencer [19]. The seven samples included four NRC sires and three pools of old Norwegian breeds. Sequence data was aligned to the BTA_4.0 reference genome [31], using the MOSAIK software package and standard alignment parameters [32], and SNPs detection was performed with GigaBayes [33]. A total of 269 new single-nucleotide polymorphisms (SNPs) were revealed in this region and were genotyped in 768 NRC sires. The resulting dataset was joined with datasets containing additional SNPs previously genotyped in NRC by haplotyping and imputation of untyped genotypes. Imputation was facilitated both by the elevated LD in NRC and by extensive pedigree records being available [28-30,34,35]. Pedigree records improve haplotyping accuracy and thereby improve accuracy of association mapping $[22,23]$. The final imputed dataset contained genotypes for 556 SNPs in 2601 NRC sires, with typically only $1.2 \%$ of SNPs missing for each individual sire. Average distance between adjacent markers for the 556 SNPs included in this study was approximately $20 \mathrm{~kb}$, with some variation in SNP density across the $11 \mathrm{Mb}$ genomic region. The 556 SNPs are presented in Additional file 1 and pairwise LD between these SNPs are shown in Additional file 2 .

\section{Association mapping}

Association mapping was performed to map single SNPs and haplotypes associated with PY or CM in the genomic interval between 86 and $97 \mathrm{Mb}$ on BTA6. Three mastitis traits were included in the analyses; incidences of $\mathrm{CM}$ in the periparturient period of first (CM1), second (CM2), and third lactation (CM3). Haplotype blocks were defined by the algorithm developed by Gabriel et al. [36] (GAB) and by the four-gamete rule algorithm (GAM) described by Wang et al. [37], and all haplotype blocks defined by the GAB or the GAM algorithm were 
included in haplotype association mapping. Results for single-marker association mapping for CM1 and PY for the highest scoring region 88 to $94 \mathrm{Mb}$ are shown in Figure 1 and Figure 2, whereas single SNPs and haplotype blocks giving highest test scores for each of the four traits are presented in Table 1.

Highest likelihood-ratio test (LRT) scores for PY were found for the SNPs ss86217862 and ss86217864 located at positions $88.410 \mathrm{Mb}$ and $88.414 \mathrm{Mb}$. Both SNPs were positioned within the gene CSN1S2 and in complete LD with each other $\left(r^{2}=1\right)$. The most significant haplotype results for PY were detected for a GAM block in the interval 88.33 to $88.43 \mathrm{Mb}$ and a smaller GAB block lying within this interval ( 88.33 to $88.42 \mathrm{Mb}$ ). Both blocks encompassed the genes CSN2, HSTN, STATH and CSN1S2. Haplotype analyses for these two blocks did not reveal genome-wide significant test scores for any of the three mastitis traits.
Single-marker association mapping for CM1 and CM3 gave highest test scores for SNP rs42766480 at 90.07 $\mathrm{Mb}$, whereas highest test score from haplotype association mapping were found for a three-marker GAM block in the interval 90.64 to $90.67 \mathrm{Mb}$. In contrast to CM1 and CM3, single-marker association mapping for CM2 gave highest test scores for SNP ss290490281 at $89.63 \mathrm{Mb}$ and the most significant haplotype association for CM2 was for a four-marker GAM block in the interval 89.62 to $89.67 \mathrm{Mb}$. For all three mastitis traits highest test scores from both single-marker and haplotype association mapping were found within the interval 89 to $91 \mathrm{Mb}$. The SNPs that gave highest test scores for the three mastitis traits (rs42766480 and ss290490281) also gave high test scores for PY (Figure 1 and Figure 2). However, the SNPs rs42766480 and ss290490281 were not in high LD, as measured by $\mathrm{r}^{2}$, with the two SNPs that gave the highest test scores for PY in this

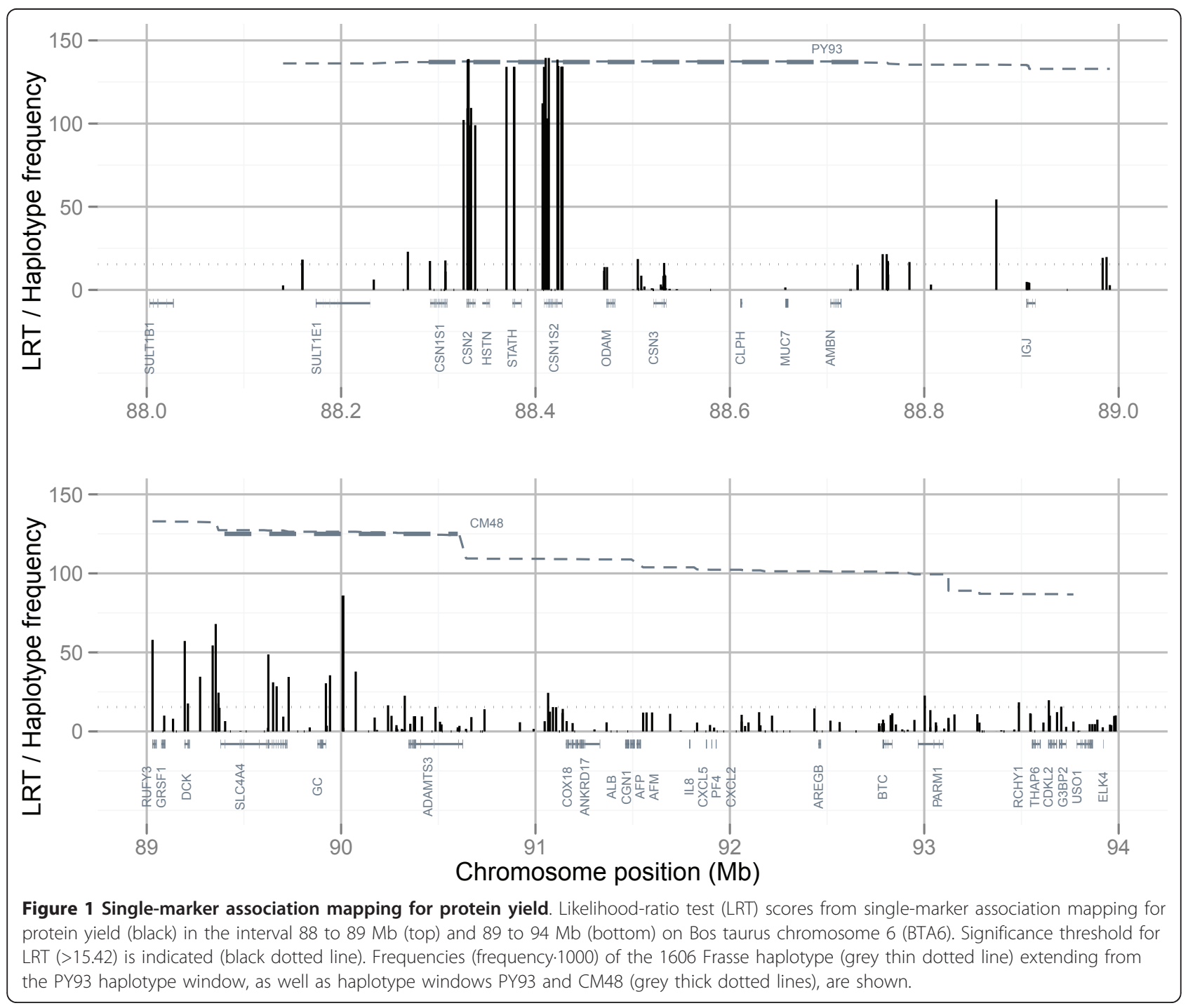






study $\left(\mathrm{r}_{\mathrm{rs} 42766480, \mathrm{ss} 86217862 / \mathrm{ss} 86217864}^{2}=0.091, \mathrm{D}_{\mathrm{rs} 42766480,}^{\prime}\right.$ $\mathrm{ss} 86217862 / \mathrm{ss} 86217864=0.734, \mathrm{r}^{2} \mathrm{ss} 290490281, \mathrm{ss} 86217862 /$

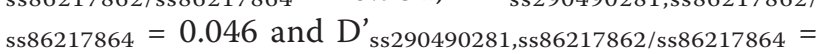
0.361). The latter indicates that the two traits PY and CM are affected by different QTLs.

\section{Molecular characterization of a long range haplotype affecting protein yield}

Very high LRT scores for PY were found for SNPs within and surrounding the casein genes CSN2 and CSN1S2 (Figure 1). A previous study using the same population also identified this region and postulated that a influential haplotype associated with elevated protein yield was introduced into NRC through importation of the bull 1606 Frasse in the 1970s [7]. To identify possible causal polymorphisms underlying this QTL whole genome re-sequencing was conducted of five elite sires in the NRC population including 1606 Frasse $(10 \times$ coverage) and two of his sons; 1893 Rud and 2005 Smidesang (both at $4 \times$ coverage).

A comparison of the sequence data covering the genes CSN2 and CSN1S2 and their 2000 bp 5'-flanking promoters with 28 previously genotyped SNPs within these regions showed that only one SNP out of the 28 SNPs was undetected by the re-sequencing. Altogether 93 polymorphisms were detected in the re-sequencing of these two regions, corresponding to one SNP approximately every $360 \mathrm{bp}$.

To be able to group genetically similar sires for the QTL for PY a 93 marker haplotype window (PY93) covering the region from 88.29 to $88.75 \mathrm{Mb}$ was defined (Figure 1 and Figure 2). Haplotype classification within PY93 was performed based on phased chromosomes for the five re-sequenced sires (Table 2) and other NRC 
Table 1 Association mapping results

\begin{tabular}{|c|c|c|c|c|}
\hline Trait & Analysis & Position (bp) & $\begin{array}{l}\text { LRT } \\
\text { score }\end{array}$ & SNPs \\
\hline $\mathrm{CM1}$ & SM & $90,075,263$ & 42 & rs42766480 \\
\hline $\mathrm{CM} 1$ & $\mathrm{GAB}$ & $\begin{array}{l}90,670,190- \\
90,725,368\end{array}$ & 37 & $\begin{array}{l}\text { ss } 61522200 \text { to } \\
\text { ss } 290490303\end{array}$ \\
\hline $\mathrm{CM} 1$ & GAM & $\begin{array}{l}90,642,598- \\
90,670,190\end{array}$ & 42 & $\begin{array}{l}\text { rs29024027 to } \\
\text { ss61522200 }\end{array}$ \\
\hline $\mathrm{CM} 2$ & SM & $89,625,793$ & 26 & ss290490281 \\
\hline $\mathrm{CM} 2$ & $\mathrm{GAB}$ & $\begin{array}{l}89,623,896- \\
89,625,793\end{array}$ & 23 & $\begin{array}{l}\text { ss61524338 to } \\
\text { ss290490281 }\end{array}$ \\
\hline $\mathrm{CM} 2$ & GAM & $\begin{array}{l}89,623,896- \\
89,668,440\end{array}$ & 28 & $\begin{array}{l}\text { ss61524338 to } \\
\text { ss86278591 }\end{array}$ \\
\hline $\mathrm{CM} 3$ & SM & $90,075,263$ & 26 & rs42766480 \\
\hline $\mathrm{CM} 3$ & GAB & $\begin{array}{l}90,670,190- \\
90,725,368\end{array}$ & 18 & $\begin{array}{l}\text { ss61522200 to } \\
\text { ss } 290490303\end{array}$ \\
\hline $\mathrm{CM} 3$ & GAM & $\begin{array}{l}90,642,598- \\
90,670,190\end{array}$ & 19 & $\begin{array}{l}\text { rs29024027 to } \\
\text { ss61522200 }\end{array}$ \\
\hline PY & SM & $88,410,501$ & 139 & ss86217862 \\
\hline PY & SM & $88,413,712$ & 139 & ss86217864 \\
\hline PY & GAB & $\begin{array}{l}88,333,706- \\
88,422,590\end{array}$ & 78 & $\begin{array}{l}\text { ss86217849 to } \\
\text { ss86217869 }\end{array}$ \\
\hline PY & GAM & $\begin{array}{l}88,333,706- \\
88,427,761\end{array}$ & 78 & $\begin{array}{l}\text { ss86217849 to } \\
\text { ss117968525 }\end{array}$ \\
\hline
\end{tabular}

Highest test scores from single-marker (SM) and haplotype (GAB and GAM) association mapping for protein yield (PY) and clinical mastitis in the periparturient period of first (CM1), second (CM2) and third lactation (CM3). Trait, analysis, chromosome position, Likelihood-ratio test (LRT) score and SNPs are presented for the highest scoring SNP or haplotype block from each analysis.

sires for which genotypes were available. The haplotypes identified in re-sequenced sires were denoted PY93_A, PY93_B, PY93_C, PY93_D and PY93_E, where PY93_A had the highest frequency and PY93_D the lowest. Each of these five haplotypes within the PY93 haplotype window was characterized by its unique set of alleles for the 93 markers. Both re-sequencing and haplotype classification indicated that 1606 Frasse was homozygous for the haplotype PY93_C, while his sons 1893 Rud and 2005 Smidesang both were heterozygous with one copy of this haplotype each. Among the 2601 sires for which genotypes and phenotypes were available, sires with at least one copy of the haplotype PY93_C, likely descendents from 1606 Frasse, had daughters with significantly elevated PY compared with remaining genotyped sires (two sample t-test gave p-value of $2.87 \mathrm{E}^{-5}$ after correcting phenotypic values for relationship matrix and grandsire effect). The haplotype PY93_C was the only haplotype found in the re-sequenced sires that had a significant association with elevated PY (p-value $<0.01$ ), motivating further characterization of this haplotype by use of re-sequencing data.

Comparison of the DNA sequence of the haplotype PY93_C with the DNA sequence of other haplotypes found in the re-sequenced sires revealed 6 polymorphisms in coding regions of casein genes CSN2 and CSN1S2 or in their 2000 bp 5'-flanking promoter regions (Table 3 ). Positive and negative alleles were assigned for these polymorphisms, with a positive allele defined as one found in PY93_C and therefore associated with increased PY (Table 3 ). The only non-synonymous substitution detected within the genes CSN2 and CSN1S2 was in amino acid 82 in CSN2 (Ref NM_181008.2), previously reported in NRC by Nilsen et al. [7]. This substitution has been reported to have differing effects on PY in various cattle breeds and is therefore not likely to be a significant causal polymorphism [8,38-40]. The re-sequencing also detected a silent substitution $(\mathrm{C}>\mathrm{T}$ ) in amino acid 125 of CSN2 (Ref NM_181008.2), previously reported by Lien et al. [5].

Of greater interest, a SNP $(\mathrm{A}>\mathrm{C})$ was detected in the promoter region of $C S N 1 S 2$ at -7 bp relative to the transcription initiation site. The SNP, which has previously been reported by Schild and Geldermann [14], was positioned three base-pairs downstream of a CCAAT motif stretching from -14 to $-10 \mathrm{bp}$. In the mammary gland the transcription factor C/EBP $\beta$ functions as an enhancer of transcription by binding to CCAAT motifs and is crucial for transcription of casein genes [41-43]. It is possible that the polymorphism described here affects the binding affinity of enhancers to the CCAAT motif, and thereby affect transcription efficiency of CSN1S2.

A second SNP $(\mathrm{T}>\mathrm{C})$, that has not previously been reported, was detected in the 5'- UTR of the gene CSN1S2 at position -5 bp distant from the initiation

Table 2 Haplotype classification for the PY93 haplotype window

\begin{tabular}{llll}
\hline Animal & Haplotype 1 (PF) & PY (p-value) & Haplotype 2 (PF) \\
\hline 1606 Frasse & PY93_C $(0.137)$ & $E\left(2.87^{-5}\right)$ & PY93_C $(0.137)$ \\
1893 Rud & PY93_C $(0.137)$ & $E\left(2.87^{-5}\right)$ & PY93_D (0.070) \\
2005 Smidesang & PY93_C $(0.137)$ & $E\left(2.87^{-5}\right)$ & PY93_B $(0.146)$ \\
2636 Vik & PY93_B $(0.146)$ & R $\left(5.35^{-13}\right)$ & PY93_E $(0.025)$ \\
3454 J. Steinsvik & PY93_A $(0.197)$ & - & PY93_E $(0.025)$
\end{tabular}

Haplotype 1 and haplotype 2 are given for NRC animals 1606 Frasse, 1893 Rud, 2005 Smidesang, 2636 Vik and 3454 J. Steinsvik for the PY93 haplotype window. Population frequencies (PF) were found based on phased chromosomes of 2601 NRC animals. Elevated (E) or reduced (R) protein yield (PY) is indicated when found significantly differing from the population mean ( $p$-value $<0.01)$. 
Table 3 Polymorphisms associated with protein yield

\begin{tabular}{lllllll}
\hline Btau_4.0 (bp) & Gene & Region & TPP * $^{*}$ & NT [+/-] & TP [+/-] & PP (bp) \\
\hline 88331023 & CSN2 & Exon & 125 & {$[\mathrm{~T} / \mathrm{C}]^{\dagger}$} & $\mathrm{P}$ & \\
88331153 & CSN2 & Exon & 82 & {$[\mathrm{C} / \mathrm{A}]^{\ddagger}$} & {$[\mathrm{P} / \mathrm{H}]$} & \\
88338754 & CSN2 & Promoter & & {$[\mathrm{G} / \mathrm{A}]$} & & -367 \\
88339252 & CSN2 & Promoter & {$[\mathrm{T} / \mathrm{A}]$} & -865 \\
88409015 & CSN1S2 & Promoter & {$[\mathrm{C} / \mathrm{A}]$} & -7 \\
88410860 & CSN1S2 & UTR & & {$[\mathrm{C} / \mathrm{T}]$} & & \\
\hline
\end{tabular}

Btau_4.0 position [31], gene, description of region (promoter, exon or UTR), translated protein position (TPP), polymorphic nucleotide (NT) alleles denoted as positive or negative [+/-], polymorphic translated protein (TP) alleles denoted as positive or negative [+/-] and promoter position (PP) relative to transcription initiation site. * Translated protein position in Ref NM_181008.2 for CSN2 and Ref NM_ 174528.2 for CSN1S2. † Polymorphism previously described in Lien et al. [5]. ¥Polymorphism previously described in Nilsen et al. [7].

codon in the sequence GYAAACatgG (Figure 3), and could directly influence translation of $\alpha_{\mathrm{S} 2}$-casein coded for by CSN1S2. Bevilacqua et al. [44] found that while transcripts from all four casein genes are found at similar concentrations in mammary tissue, translated $\alpha_{\mathrm{S}^{-}}$ casein and $\kappa$-casein are found in much lower concentrations in cow milk than $\alpha_{\mathrm{S1}}$-casein and $\beta$-casein. The 5'UTR sequence for the four caseins were strictly conserved between cattle, sheep and goat, and they suggested that variation in the Kozak consensus sequence (GCCRCCatgG [45]) might be the cause of the observed variation in translational efficiency between casein genes (Figure 3). Matching well with the higher protein levels associated with the PY93_C haplotype from 1606 Frasse; the $\mathrm{C}$ allele found in PY93_C was in better accordance with the Kozak consensus sequence than the alternative

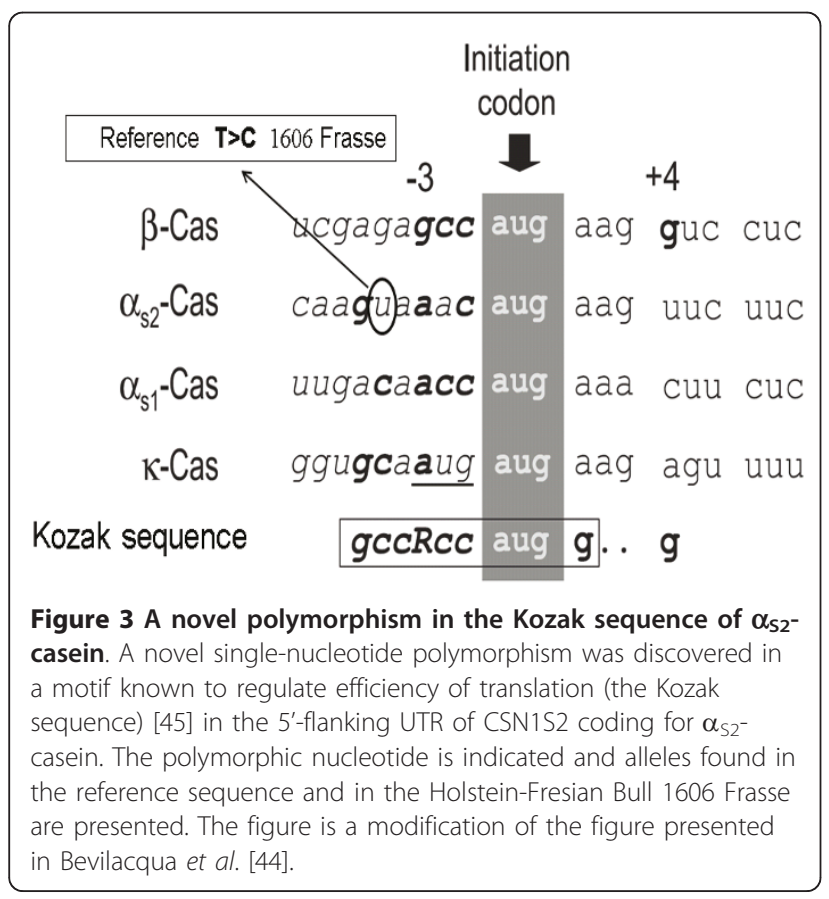

$\mathrm{T}$ allele, and therefore expected to produce a more efficient translation initiation site within the CSN1S2 transcript. Work is in progress to deduce functionality of the detected polymorphisms on the transcription and translation of CSN1S2 by expression profiling and quantitative determination of $\alpha_{\mathrm{S} 2}$-casein in milk.

\section{Long range haplotypes affecting clinical mastitis}

Highest test scores for CM1, CM2 and CM3 from both single-marker and haplotype association mapping were found within the genomic interval 89 to $91 \mathrm{Mb}$ on BTA6 (Table 1). To be able to group genetically similar sires for the QTL for CM a 48 marker haplotype window (CM48) covering the genomic interval 89.4 to 90.6 $\mathrm{Mb}$ was defined (Figure 1 and Figure 2). This interval contained the SNPs producing the highest test scores from association mapping for CM. Haplotype classification within CM48 was performed based on phased chromosomes for NRC sires for which genotypes were available. The sire 1606 Frasse was homozygous for the CM48 haplotype window, and sires with at least one copy of the haplotype found in 1606 Frasse had daughters with significantly elevated mastitis susceptibility compared with remaining genotyped sires (two sample $\mathrm{t}$-test gave $\mathrm{p}$-value of $2.03 \mathrm{E}^{-5}$ after correcting phenotypic values for relationship matrix and grandsire effect).

As can be seen from Figure 1 and Figure 2, the 1606 Frasse haplotype was not markedly disrupted by recombination between the haplotype windows PY93 and CM48. This means that although there were no high LD between SNPs showing high association with PY and SNPs showing high association with CM in the genotyped population as a whole, there were strong correlations between these SNPs within individuals holding a copy of the haplotype from Frasse 1606 for this region. As previously noted the haplotype from Frasse 1606 was also significantly associated with higher levels of protein content in milk [5,7], which might partly explain the observed genetic correlation between PY and $\mathrm{CM}$ in NRC [1].

In contrast to the QTL for PY which gave very high test scores for potent candidate genes (Figure 1), results for CM were much more dispersed (Figure 2). SNPs strongly associated with $\mathrm{CM}$ were not concentrated to a few specific genes, meaning that a number of genes in the QTL region for CM could harbor polymorphisms potentially affecting mastitis susceptibility. Previously the genes MUC7 and IGJ have been proposed as candidate genes for mastitis susceptibility in this region [6], but elevated test scores for CM were not found in or around these two genes in the current study (Figure 2).

The highest test scores for $\mathrm{CM}$ were found within and surrounding the three genes SLC4A4, GC and ADAMTS3. The first of these $(S L C 4 A 4)$ codes for a 
sodium bicarbonate co-transporter involved in maintaining normal blood pH $[46,47]$, the second $(G C)$ encodes the main carrier protein of vitamin $\mathrm{D}$ in plasma, and finally the third gene (ADAMTS3) shows high similarity with $A D A M T S 2$, which codes for a pro-collagen N-proteinase [48]. A cluster of genes coding for the CXC chemokines IL8, CXCL5, PF4 and CXCL2 are positioned around $92 \mathrm{Mb}$, quite close to the highest scoring region for CM. CXC chemokines are important pro-inflammatory mediators and might therefore contain variation affecting mastitis susceptibility. Expression levels of some of these CXC chemokines have been shown to increase in bovine mammary gland cell lines after exposure to bacterial cell wall components [49-52], underlining their relevance as candidate genes in this QTL region.

\section{Conclusion}

Highest test scores from association mapping for PY were found within and surrounding the casein genes CSN2 and CSN1S2. Haplotype classification and highcoverage re-sequencing data indicated that the HolsteinFriesian bull 1606 Frasse, imported into the NRC cattle population in the 1970s, was homozygous for a haplotype encompassing these two genes. As previously suggested the haplotype from 1606 Frasse was significantly associated with elevated PY and selection for milk production traits is likely to have increased the frequency of this haplotype in the NRC population [5]. Data available from high throughput re-sequencing allowed for molecular characterization of the haplotype from 1606 Frasse, and plausible causal polymorphisms were detected in a regulatory element in the promoter region of the gene CSN1S2 as well as in a motif that regulates translation efficiency of CSN1S2 [44,45].

It was further shown that the long range haplotype from 1606 Frasse is highly conserved in the NRC population for the region spanning the two QTLs affecting PY and CM on BTA6. The positive effect on milk protein content and the negative effect on mastitis susceptibility of this haplotype might partly explain the observed genetic correlation between these two traits in NRC [1]. Our results emphasize the importance of inclusion of mammary gland health in dairy cattle breeding goals.

\section{Methods}

\section{Animals and phenotypes}

NRC is an admixed breed formed from Norwegian breeds and imported animals from other Nordic countries. Norway has a dairy herd recording system which has included veterinary reported clinical mastitis (VRCM) since 1975 [53]. Records of VRCM in the periparturient period ( -15 to 30 days post partum) of first
(CM1), second (CM2) and third (CM3) lactation were available from GENO Breeding and AI Association [54] as daughter-yield-deviations (DYDs) for NRC sires. DYDs are phenotypic values corrected for non-genetic effects believed to affect the trait, and a sire with a high DYD value for CM has daughters with increased susceptibility to CM. Here records of VRCM were retrieved as a binary trait for daughters of sires from paternal half-sib families, yielding a large number of records per sire and a reduction in variance due to environmental effects compared with other designs [55]. The mastitis traits CM1, CM2 and CM3 are described in Table 4 together with heritabilities and genetic correlations reported by Svendsen and Heringstad [54].

Records of PY were also available from GENO Breeding and AI Association as DYDs for NRC sires and were retrieved as 3,481,538 daughter records of 2,596 sires from paternal half-sib families. A sire with a high DYD value for PY has daughters with increased PY. For both traits (CM and PY) number of daughter records per sire was highly variable and influenced by a small number of elite sires with a large number of daughters. DNA samples for sires included in this study were extracted from semen available from GENO Breeding and AI Association.

\section{Re-sequencing of candidate region and SNP detection}

Sequence capture using a Roche-Nimblegen product was performed to isolate the region of interest on BTA6. Roche NimbleGen designed and manufactured a $5 \mathrm{Mb}$ sequence capture array targeting BTA6 coordinates 88$97 \mathrm{Mb}$ (385 K NimbleGen sequence capture arrays), standard repeat masking (approximately $50 \%$ of the sequence was masked) was applied in the design with $80 \%$ of the targeted bases being within a 100 bp window of the final probe set. Sequence capture library construction was performed on seven samples, four NRC sires (2005 Smidesang, 10243 Rishaugen, 10263 Frestad and 10553 Nordbø) and three pools of old Norwegian breeds. Samples were sequenced using a 454 GS-FLX platform with the number of reads generated from each sample ranging from 97-460 k. Sequence data from all samples was aligned to the BTA_4.0 reference genome

Table 4 The mastitis traits

\begin{tabular}{cccccc}
\hline & \multicolumn{4}{c}{ Number of records } & \multicolumn{4}{c}{ Heritability and genetic correlation } \\
Trait & Daughters & Sires & CM1 & CM2 & CM3 \\
\hline CM1 & $1,755,649$ & 2,596 & 0.03 & 0.74 & 0.68 \\
CM2 & $1,256,887$ & 2,532 & - & 0.02 & 0.85 \\
CM3 & 805,376 & 2,440 & - & - & 0.02 \\
\hline
\end{tabular}

Trait and number of sire and daughter records for the clinical mastitis traits CM1, CM2 and CM3 are presented. The final columns give heritabilities (on the diagonal) and genetic correlations reported by Svendsen and Heringstad [54]. 
[31] using the MOSAIK software package and default alignment parameters [32], yielding an average coverage of $5 \times$ for the region of interest.

SNP detection was performed with GigaBayes [33]. Criteria for filtering SNPs included minimum number of reads of each variant in non-coding regions $(\geq 2)$, minimum number of reads of the variant differing from the reference sequence for coding region $(\geq 2)$, GigaBayes score $(\geq 0.95)$ and minimum distance to closest SNP $(>5$ bp). SNPs positioned in homopolymer regions $(>5 \mathrm{bp})$ were also rejected. After filtering 269 SNPs remained.

\section{Genotype datasets}

Four genotype datasets were combined to yield a final dataset of 556 SNPs. The 269 SNPs identified by resequencing of the candidate region by Roche NimbleGen sequence capture and 454 GS-FLX sequencing were genotyped in 768 sires from paternal half-sib families using the Sequenom MassARRAY system (dataset 1). Genotypes were also retrieved for 84 SNPs for 2589 NRC sires from paternal half-sib families genotyped with the Affymetrix $25 \mathrm{k}$ MIP array (dataset 2) and for 198 SNPs for 1092 NRC sires from paternal half-sib families genotyped with the Illumina Bovine SNP50 BeadChip (dataset 3). Finally, genotypes for 102 SNPs in the genomic region 86-90.4 Mb on BTA6 were retrieved for 1133 sires [6] (dataset 4). Minimum and maximum number of SNPs a sire could be genotyped for were 84 and 556, respectively. Some SNPs were present in more than one dataset (table 5).

\section{Data correction and imputation}

The genotyped data were checked for mendelian errors and based on the observed results, a cut-off of $4 \%$ was set to identify samples not fitting the pedigree. Pedigree errors were resolved by either identifying a new sire or setting parental information to unknown for the affected animal. New sires were assigned when the number of mendelian errors was equal to or lower than the background $(0.4 \%)$ and there was only one candidate.

Linkage analysis, haplotyping and imputation were conducted with CRIMAP [56] and locally developed software to combine the four datasets and fill in untyped

\section{Table 5 Genotype datasets}

\begin{tabular}{lllll}
\hline Dataset & Source & SNPs & Unique SNPs & Sires \\
\hline 1 & 454 re-sequencing & 269 & 269 & 768 \\
2 & $25 \mathrm{k}$ & 84 & 35 & 2589 \\
3 & $50 \mathrm{k}$ & 198 & 121 & 1092 \\
4 & Nilsen et al., 2009 [7] & 103 & 96 & 1133 \\
\hline
\end{tabular}

The four genotype datasets that contributed to the final genotype dataset of 556 SNPs used for association mapping are summarized. For each dataset

source of SNPs, number of SNPs used, number of SNPs unique for the dataset and number of sires genotyped for the SNPs are given. genotypes. The phasing procedure was implemented based on the six rules algorithm presented by Qian et al. [57], with modifications to fit half-sib families with missing data. Imputation in paternal haplotypes was performed by assuming no recombination between informative markers of the same phase. Imputation in haplotypes where no parental genotype information was available was performed by searching the rest of the dataset for equal haplotypes at surrounding informative positions and imputing when the untyped genotype could be decided uniquely. The final dataset contained genotypes for 556 SNPs in the BTA6 86-97 Mb genomic region for 2601 sires, with on average 1.2\% of SNPs missing for each individual sire.

\section{Single-marker association mapping}

Single-marker association mapping for CM1, CM2, CM3 and PY were performed for all SNPs. The mixed model was:

$$
P_{i}=X g_{j}+Y a_{i}+Z m_{k}+e_{i j k}
$$

Here phenotypic value P is DYD of sire i weighted by number of daughters, $g$ is fixed effect of grandsire $j$, $a$ is random effect of sire i where co-variance structure between sires is determined from pedigree relationships, $m$ is random effect of genetic marker $k$ and $e$ is an error term. Estimation was conducted with the ASREML software [58].

\section{Haplotype association mapping}

Pair-wise LD measure $\mathrm{r}^{2}$ was found for all SNP pairs with the Haploview 4.1 software [59] and haplotype blocks were defined by the method described in Gabriel et al. [36] (GAB) and by the four gamete rule [37] (GAM). A perl script was written to classify sires according to haplotypes for each of the defined haplotype blocks. The classification into GAB and GAM blocks were implemented in haplotype association mapping for CM1, CM2, CM3 and PY. The mixed model was:

$$
P=X g_{i}+Y a_{j}+Z h_{k}+e_{i j k}
$$

Here phenotypic value P is DYD of sire i weighted by number of daughters, $g$ is fixed effect of grandsire $j, a$ is random effect of sire i where co-variance structure between sires is determined from pedigree relationships, $h$ is random effect of haplotype $k$ and $e$ is an error term. Estimation was conducted with the ASREML software [58].

\section{Test score and multiple testing}

LRT scores were calculated as two times the log-likelihood ( $\log \mathrm{L})$ ratio. $\log \mathrm{L}$ ratios were obtained with the 
ASREML software [58] for each SNP or haplotype as the difference between the LogL of a model containing the effect of the SNP or haplotype and the LogL of a model not containing this effect. LRT scores were expected to be distributed as a mixture of two $\chi^{2}$ distributions with 0 and 1 degree of freedom. To correct for multiple testing the significance threshold was corrected with the effective number of independent tests $\left(\mathrm{Meff}_{\mathrm{G}}\right)$ [60]. $\mathrm{Meff}_{G}$ was found to be 208 for the 556 genotyped SNPs, corresponding to an adjusted p-value of $4.8 \mathrm{E}^{-5}(0.01 / 208$ $=4.8 \mathrm{E}^{-5}$ ) and a LRT score of 15.42 .

MacLeod et al. [61] demonstrated that including effect of sire based on pedigree relationships reduces the number of false positives in association studies.

\section{Genome re-sequencing and detection of polymorphisms} Genome re-sequencing of five NRC sires (1606 Frasse, 2636 Vik, 3454 J. Steinsvik, 2005 Smidesang and 1893 Rud) was performed on an Illumina GAIIx platform in order to characterize the haplotypes of these animals and identify putative causal polymorphims. Reads were generated as $2 \times 108$ paired-ends, coverage was $10 \mathrm{~Gb}$ for 1606 Frasse and $2636 \mathrm{Vik}$, and $4 \mathrm{~Gb}$ for $3454 \mathrm{~J}$. Steinsvik, 2005 Smidesang and 1893 Rud. The FASTQ/ A Clipper program from the FASTX-Toolkit [62] was used to remove adapter sequence and to discard reads based on average quality score $(<10)$ or untyped bases ( $>7 \mathrm{Ns}$ ). Sequence data was assembled by mapping reads to the BTA_4.0 reference genome [31] using the BWA software package [63] and dafault alignment parameters. Polymorphism detection was performed with SAMtools [64]. Criteria for filtering included minimum number of reads $(\geq 2)$, maximum number of reads $(\leq 100)$, minimum number of reads of the variant differing from the reference sequence $(\geq 2)$ and minimum RMS mapping value $(\geq 25)$. Polymorphisms positioned in homopolymer or repeat regions were discharged.

\section{Additional material}

Additional file 1: Genotyped single-nucleotide polymorphisms. The 556 single nucleotide polymorphisms (SNPs) genotyped in this study are presented by position, alleles and missing genotype percentage.

Additional file 2: Linkage disequilibrium. Pairwise linkage disequilibrium (LD) between all pairs of the 556 single nucleotide polymorphisms (SNPs) genotyped in this study presented by Bos taurus chromosome 6 (BTA6) positions (complete LD is indicated in white).

\section{Acknowledgements}

Thanks to Paul R. Berg, Marianne H. S. Hansen, Hanne Hamland, Arne Roseth and Kristil K. Sundsaasen for sample processing, to Tina Graceline for bioinformatics assistance and to GENO Breeding and Al association for providing relationship information and DYD values. This project has been funded by The Research Council of Norway, GENO Breeding and Al association and BoviBank Ltd.

\section{Author details}

${ }^{1}$ Centre for Integrative Genetics, Department of Animal and Aquacultural Sciences, Norwegian University of Life Sciences, N-1432 Aas, Norway. ${ }^{2}$ Geno Breeding and Al organization, Norwegian University of Life Sciences, Box 5003, N-1432 Aas, Norway. ${ }^{3}$ Biosciences Research Division, Department of Primary Industries Victoria, Melbourne, Australia, 3083.

\section{Authors' contributions}

MS bioinformatics on 454 sequence data, statistical analyses and writing the manuscript. HG joining datasets by haplotyping and imputation. MK conducted and coordinated molecular genetics work. ST bioinformatics on Illumina sequence data. MS provided DYDs and pedigree information. BJH assisted in drafting the manuscript. SL conceived of and coordinated the study and assisted in drafting the manuscript. All authors helped finalize the manuscript and read and approved of the final version. The authors declare that they have no competing interests.

Received: 6 February 2011 Accepted: 11 August 2011 Published: 11 August 2011

\section{References}

1. Heringstad B, Chang YM, Gianola D, Klemetsdal G: Genetic association between susceptibility to clinical mastitis and protein yield in norwegian dairy cattle. J Dairy Sci 2005, 88(4):1509-1514

2. Heringstad B, Klemetsdal G, Ruane J: Selection for mastitis resistance in dairy cattle: a review with focus on the situation in the Nordic countries. Livestock Production Science 2000, 64(2-3):95-106.

3. Heringstad B, Chang YM, Gianola D, Klemetsdal G: Genetic analysis of clinical mastitis, milk fever, ketosis, and retained placenta in three lactations of Norwegian red cows. J Dairy Sci 2005, 88(9):3273-3281.

4. Lund MS, Jensen J, Petersen PH: Estimation of genetic and phenotypic parameters for clinical mastitis, somatic cell production deviance, and protein yield in dairy cattle using Gibbs sampling. J Dairy Sci 1999, 82(5):1045-1051.

5. Lien S, Gomez-Raya L, Steine T, Fimland E, Rogne S: Associations between casein haplotypes and milk yield traits. J Dairy Sci 1995, 78(9):2047-2056.

6. Nilsen $H$, Olsen $H G$, Hayes $B$, Nome $T$, Sehested $E$, Svendsen $M$, Meuwissen TH, Lien S: Characterization of a QTL region affecting clinical mastitis and protein yield on BTA6. Anim Genet 2009, 40(5):701-712.

7. Nilsen $H$, Olsen $H G$, Hayes B, Sehested $E$, Svendsen M, Nome T, Meuwissen T, Lien S: Casein haplotypes and their association with milk production traits in Norwegian Red cattle. Genet Sel Evol 2009, 41:24.

8. Bovenhuis $\mathrm{H}$, Weller Jl: Mapping and analysis of dairy cattle quantitative trait loci by maximum likelihood methodology using milk protein genes as genetic markers. Genetics 1994, 137(1):267-280.

9. Velmala RJ, Vilkki HJ, Elo KT, de Koning DJ, Maki-Tanila AV: A search for quantitative trait loci for milk production traits on chromosome 6 in Finnish Ayrshire cattle. Anim Genet 1999, 30(2):136-143.

10. Schopen GC, Koks PD, van Arendonk JA, Bovenhuis H, Visker MH: Whole genome scan to detect quantitative trait loci for bovine milk protein composition. Anim Genet 2009, 40(4):524-537.

11. Farrell HM Jr, Jimenez-Flores R, Bleck GT, Brown EM, Butler JE, Creamer LK, Hicks CL, Hollar CM, Ng-Kwai-Hang KF, Swaisgood HE: Nomenclature of the proteins of cows' milk-sixth revision. J Dairy Sci 2004, 87(6):1641-1674

12. Martin P, Szymanowska M, Zwierzchowski L, Leroux C: The impact of genetic polymorphisms on the protein composition of ruminant milks. Reprod Nutr Dev 2002, 42(5):433-459.

13. Caroli AM, Chessa S, Erhardt GJ: Invited review: milk protein polymorphisms in cattle: effect on animal breeding and human nutrition. J Dairy Sci 2009, 92(11):5335-5352.

14. Schild TA, Geldermann H: Variants within the $5^{\prime}$-flanking regions of bovine milk-protein-encoding genes. III. Genes encoding the Casensitive caseins as1, as2 and $\beta$. Theoretical and Applied Genetics 1996, 93:887-893.

15. Hallen E, Wedholm A, Andren A, Lunden A: Effect of beta-casein, kappacasein and beta-lactoglobulin genotypes on concentration of milk protein variants. J Anim Breed Genet 2008, 125(2):119-129.

16. Szymanowska M, Siadkowska E, Lukaszewicz M, Zwierzchowski L: Association of nucleotide-sequence polymorphism in the $5^{\prime}$-flanking 
regions of bovine casein genes with casein content in cow's milk. Le Lait 2004, 84:579-590.

17. Mardis ER: The impact of next-generation sequencing technology on genetics. Trends Genet 2008, 24(3):133-141.

18. Stratton M: Genome resequencing and genetic variation. Nat Biotechnol 2008, 26(1):65-66.

19. Albert TJ, Molla MN, Muzny DM, Nazareth $L$, Wheeler D, Song $X$, Richmond TA, Middle CM, Rodesch MJ, Packard CJ, et al: Direct selection of human genomic loci by microarray hybridization. Nat Methods 2007, 4(11):903-905

20. Weaver TA: High-throughput SNP discovery and typing for genome-wide genetic analysis. Trends in Genetics 2000, 2000:36-42

21. Vignal A, Milan D, SanCristobal M, Eggen A: A review on SNP and other types of molecular markers and their use in animal genetics. Genet Sel Evol 2002, 34(3):275-305.

22. Lindholm E, Zhang J, Hodge SE, Greenberg DA: The reliability of haplotyping inference in nuclear families: misassignment rates for SNPs and microsatellites. Hum Hered 2004, 57(3):117-127.

23. Gao G, Allison DB, Hoeschele I: Haplotyping methods for pedigrees. Hum Hered 2009, 67(4):248-266.

24. Nothnagel M, Ellinghaus D, Schreiber S, Krawczak M, Franke A: A comprehensive evaluation of SNP genotype imputation. Hum Genet 2009, 125(2):163-171.

25. Hao K, Chudin E, McElwee J, Schadt EE: Accuracy of genome-wide imputation of untyped markers and impacts on statistical power for association studies. BMC Genet 2009, 10:27.

26. Pei YF, Zhang L, Li J, Deng HW: Analyses and comparison of imputationbased association methods. PLoS One 2010, 5(5):e10827.

27. Browning SR: Missing data imputation and haplotype phase inference for genome-wide association studies. Hum Genet 2008, 124(5):439-450.

28. Vallejo RL, Li YL, Rogers GW, Ashwell MS: Genetic diversity and background linkage disequilibrium in the North American Holstein cattle population. J Dairy Sci 2003, 86(12):4137-4147

29. Odani M, Narita A, Watanabe T, Yokouchi K, Sugimoto Y, Fujita T, Oguni T, Matsumoto M, Sasaki Y: Genome-wide linkage disequilibrium in two Japanese beef cattle breeds. Anim Genet 2006, 37(2):139-144.

30. Khatkar MS, Nicholas FW, Collins AR, Zenger KR, Cavanagh JA, Barris W, Schnabel RD, Taylor JF, Raadsma HW: Extent of genome-wide linkage disequilibrium in Australian Holstein-Friesian cattle based on a highdensity SNP panel. BMC Genomics 2008, 9:187.

31. Liu $Y$, Qin $X$, Song $X Z$, Jiang $H$, Shen $Y$, Durbin $K$, Lien S, Kent MP, Sodeland $M$, Ren $Y$, et al: Bos taurus genome assembly. BMC Genomics 2009, 10:180.

32. Strömberg M: Mosaik 1.0 Documentation. 2009

33. Marth GT: GigaBayes: SNP and Short-INDEL Polymorphism Discovery Tool. 2009.

34. Farnir F, Coppieters W, Arranz JJ, Berzi P, Cambisano N, Grisart B, Karim L, Marcq F, Moreau L, Mni M, et al: Extensive genome-wide linkage disequilibrium in cattle. Genome Res 2000, 10(2):220-227.

35. Tenesa A, Knott SA, Ward D, Smith D, Williams JL, Visscher PM: Estimation of linkage disequilibrium in a sample of the United Kingdom dairy cattle population using unphased genotypes. J Anim Sci 2003, 81(3):617-623.

36. Gabriel SB, Schaffner SF, Nguyen H, Moore JM, Roy J, Blumenstiel B, Higgins J, Defelice M, Lochner A, Faggart $M$, et al: The structure of haplotype blocks in the human genome. Science 2002, 296(5576):2225-2229.

37. Wang N, Akey JM, Zhang K, Chakraborty R, Jin L: Distribution of recombination crossovers and the origin of haplotype blocks: the interplay of population history, recombination, and mutation. Am J Hum Genet 2002, 71(5):1227-1234.

38. Boettcher PJ, Caroli A, Stella A, Chessa S, Budelli E, Canavesi F, Ghiroldi S, Pagnacco G: Effects of casein haplotypes on milk production traits in Italian Holstein and Brown Swiss cattle. J Dairy Sci 2004, 87(12):4311-4317.

39. Ikonen T, Bovenhuis H, Ojala M, Ruottinen O, Georges M: Associations between casein haplotypes and first lactation milk production traits in Finnish Ayrshire cows. J Dairy Sci 2001, 84(2):507-514.

40. Velmala R, Vilkki J, Elo K, Maki-Tanila A: Casein haplotypes and their association with milk production traits in the Finnish Ayrshire cattle. Anim Genet 1995, 26(6):419-425

41. Wyszomierski SL, Rosen JM: Cooperative effects of STAT5 (signal transducer and activator of transcription 5) and C/EBPbeta (CCAAT/ enhancer-binding protein-beta) on beta-casein gene transcription are mediated by the glucocorticoid receptor. Mol Endocrinol 2001, 15(2):228-240.

42. Robinson GW, Johnson PF, Hennighausen L, Sterneck E: The C/EBPbeta transcription factor regulates epithelial cell proliferation and differentiation in the mammary gland. Genes Dev 1998, 12(12):1907-1916

43. Rosen JM, Wyszomierski SL, Hadsell D: Regulation of milk protein gene expression. Annu Rev Nutr 1999, 19:407-436.

44. Bevilacqua C, Helbling JC, Miranda G, Martin P: Translational efficiency of casein transcripts in the mammary tissue of lactating ruminants. Reprod Nutr Dev 2006, 46(5):567-578.

45. Kozak M: Recognition of AUG and alternative initiator codons is augmented by $\mathrm{G}$ in position +4 but is not generally affected by the nucleotides in positions +5 and +6 . EMBO J 1997, 16(9):2482-2492.

46. Yu H, Riederer B, Stieger N, Boron WF, Shull GE, Manns MP, Seidler UE, Bachmann O: Secretagogue stimulation enhances NBCe1 (electrogenic $\mathrm{Na}(+) / \mathrm{HCO}(3)(-)$ cotransporter) surface expression in murine colonic crypts. Am J Physiol Gastrointest Liver Physiol 2009, 297(6):G1223-1231.

47. Igarashi T, Sekine T, Watanabe H: Molecular basis of proximal renal tubular acidosis. J Nephrol 2002, 15(Suppl 5):S135-141.

48. Tang BL: ADAMTS: a novel family of extracellular matrix proteases. Int $J$ Biochem Cell Biol 2001, 33(1):33-44.

49. Yu C, Shi ZR, Chu CY, Lee KH, Zhao X, Lee JW: Expression of bovine granulocyte chemotactic protein-2 (GCP-2) in neutrophils and a mammary epithelial cell line (MAC-T) in response to various bacterial cell wall components. Vet J 2010, 186(1):89-95.

50. Griesbeck-Zilch B, Meyer HH, Kuhn CH, Schwerin M, Wellnitz O: Staphylococcus aureus and Escherichia coli cause deviating expression profiles of cytokines and lactoferrin messenger ribonucleic acid in mammary epithelial cells. J Dairy Sci 2008, 91(6):2215-2224.

51. McClenahan D, Krueger R, Lee HY, Thomas C, Kehrli ME Jr, Czuprynski C: Interleukin-8 expression by mammary gland endothelial and epithelial cells following experimental mastitis infection with E. coli. Comp Immunol Microbiol Infect Dis 2006, 29(2-3):127-137.

52. Mount JA, Karrow NA, Caswell JL, Boermans HJ, Leslie KE: Assessment of bovine mammary chemokine gene expression in response to lipopolysaccharide, lipotechoic acid + peptidoglycan, and CpG oligodeoxynucleotide 2135. Can J Vet Res 2009, 73(1):49-57.

53. Østeras $O$, Solbu H, Refsdal AO, Roalkvam T, Filseth O, Minsaas A: Results and evaluation of thirty years of health recordings in the Norwegian dairy cattle population. J Dairy Sci 2007, 90(9):4483-4497.

54. Svendsen M, Heringstad B: New Genetic Evaluation for Clinical Mastitis in Multiparous Norwegian Red Cows. Interbull Bulletine 2006, 35:8-11.

55. Weller Jl, Kashi Y, Soller M: Power of daughter and granddaughter designs for determining linkage between marker loci and quantitative trait loci in dairy cattle. J Dairy Sci 1990, 73(9):2525-2537.

56. Green P, Falls K, Crooks S: Documentation for CRI-MAP, version 2.4. Washington University School of Medicine, St. Louis, Mo., USA. 1990.

57. Qian D, Beckmann L: Minimum-recombinant haplotyping in pedigrees. Am J Hum Genet 2002, 70(6):1434-1445.

58. Gilmour AR, Cullis BR, Welham SJ, Thompson R: ASREML reference manual. 2000.

59. Barrett JC, Fry B, Maller J, Daly MJ: Haploview: analysis and visualization of LD and haplotype maps. Bioinformatics 2005, 21(2):263-265.

60. Gao X, Starmer J, Martin ER: A multiple testing correction method for genetic association studies using correlated single nucleotide polymorphisms. Genet Epidemiol 2008, 32(4):361-369.

61. MacLeod IM, Hayes BJ, Savin KW, Chamberlain AJ, McPartlan HC, Goddard ME: Power of a genome scan to detect and locate quantitative trait loci in cattle using dense single nucleotide polymorphisms. J Anim Breed Genet 2010, 127(2):133-142.

62. FASTX-Toolkit. [http://hannonlab.cshl.edu/fastx_toolkit/index.html].

63. Li H, Durbin R: Fast and accurate short read alignment with BurrowsWheeler transform. Bioinformatics 2009, 25(14):1754-1760.

64. Li H, Handsaker B, Wysoker A, Fennell T, Ruan J, Homer N, Marth G, Abecasis G, Durbin R: The Sequence Alignment/Map format and SAMtools. Bioinformatics 2009, 25(16):2078-2079.

doi:10.1186/1471-2156-12-70

Cite this article as: Sodeland et al:: Molecular characterization of a long range haplotype affecting protein yield and mastitis susceptibility in Norwegian Red cattle. BMC Genetics 2011 12:70. 